\title{
Estimation des expositions associées à la présence du radon 222 dans les usines de traitement de phosphates et leurs environnements en Tunisie
}

\author{
H. MAHJOUBI*, A.H. CHARFI**, S. LABIDI*, N. CHAHED*, S. MTIMET*
}

(Manuscrit reçu le 17 juin 1999, révisé le 6 décembre 1999, accepté le 15 janvier 2000)

RÉSUMÉ L'étude des expositions associées à la présence du ${ }^{222} \mathbf{R n}$ dans les usines de traitement de phosphates et leurs environnements nécessite la mise en ouvre d'un certain nombre de mesures dont les résultats devront ensuite être comparés aux recommandations de la CIPR dans sa publication 65. L'objectif de ces mesures est d'une part d'identifier les lieux qui peuvent présenter une exposition potentielle par la mesure de la concentration de radon 222 et d'autre part d'estimer cette exposition pour les travailleurs par la détermination de l'énergie alpha potentielle volumique. Le niveau de l'énergie alpha potentielle volumique à la surface du dépôt de phosphogypse «tabia» dépasse les recommandations de la CIPR dans certains points de mesure.

ABSTRACT Estimation of the exposure related to the presence of radon 222 indoor phosphate treatmen factories and their environments in Tunisia.

The study of the exposures linked to the existance of ${ }^{222} \mathrm{Rn}$ in the factories of phosphate treatment and their environment, needs implementation of some special measurements which results should be compared with ICRP recommendations. The objective of these measurements is to identify the sites that could present a potentiel exposure, by measuring radon 222 concentration. On the other hand, it is to estimate this risk by identifying the alpha potential energy. In some cases the alpha potential energy level on the surface of phosphogyps storage can exceed the ICRP recommendations.

\section{Introduction}

Les phosphates constituent la principale richesse minière de la Tunisie. Une grande partie de ce minerai est transformée en acide phosphorique et en engrais dans les usines du groupe chimique. La transformation du phosphate lavé en acide phosphorique se fait principalement par réaction de l'acide sulfurique sur le phosphate, et ce dans des réacteurs appropriés sous des conditions définies de température et de concentration en $\mathrm{P}_{2} \mathrm{O}_{5}$ et en sulfate.

* Centre national de radio-protection, Tunis, Tunisic.
** Groupe chimique tunisien, Sfax, Tunisie. 
Le produit de réaction est formé d'une phase solide composée principalement de phosphogypse en suspension dans une solution d'acide phosphorique contenant $28 \%$ de $\mathrm{P}_{2} \mathrm{O}_{5}$ est filtré sous vide. Le phosphogypse est déposé dans une aire aménagée «tabia ».

La radioactivité dans les phosphates provient des chaînes de l' ${ }^{238} \mathrm{U}$ et $d u{ }^{232} \mathrm{Th}$. Les concentrations des descendants $\mathrm{du}^{232} \mathrm{Th}$ sont souvent faibles. Par contre celles de ${ }^{\prime}{ }^{238} \mathrm{U}$ sont relativement abondantes.

Les risques radiologiques sont différents suivant le mode d'exposition au radioélément, ainsi le risque majeur lié aux radioéléments émetteurs existe lorsque il y a risque d'absorption de poussières contenant ce type de radioélément. En outre en se désintégrant le radon émet des particules alpha et engendre des descendants solides eux même radioactifs. Ces particules peuvent être fixées sur les aérosols atmosphériques et peuvent être inhalées et engendrer des effets néfastes pour la santé.

Dans cette étude nous nous sommes intéressés à la recherche des postes de travail les plus exposés ainsi qu'à l'évaluation de l'exposition radiologique due à la présence du radon 222 dans les trois usines : Sfax, Skhira, M'dhilla et dans leurs environnements afin de prévenir les risques radiologiques dans les ambiances de travail.

\section{Activité pondérale en ${ }^{226} \mathrm{Ra}$ dans les produits de traitements des phosphates}

Nous avons étudié les différents maillons de la chaîne de fabrication de l'acide phosphorique en partant de la source qui est le phosphate jusqu'au déchet de traitement qui est le phosphogypse. Le phosphogypse est stocké à l'air libre à côté des usines de traitements de phosphates et forme les « tabias » de phosphogypse (Figs. 1 à 3).

Cinq échantillons de phosphogypse et de phosphate ont été analysé par spectrométrie gamma. Le premier résultat que nous avons déduit facilement d'après nos mesures est qu'il y a une redistribution de la radioactivité et le ${ }^{226} \mathrm{Ra}$ se retrouve en majorité dans le phosphogypse (Tab. I).

\section{Dose efficace due au ${ }^{226} \mathrm{Ra}$}

Un agent de l'usine est exposé au ${ }^{226}$ Ra présent aussi bien dans les phosphates lavés (produits à traiter) que dans le phosphogypse (résidus). 


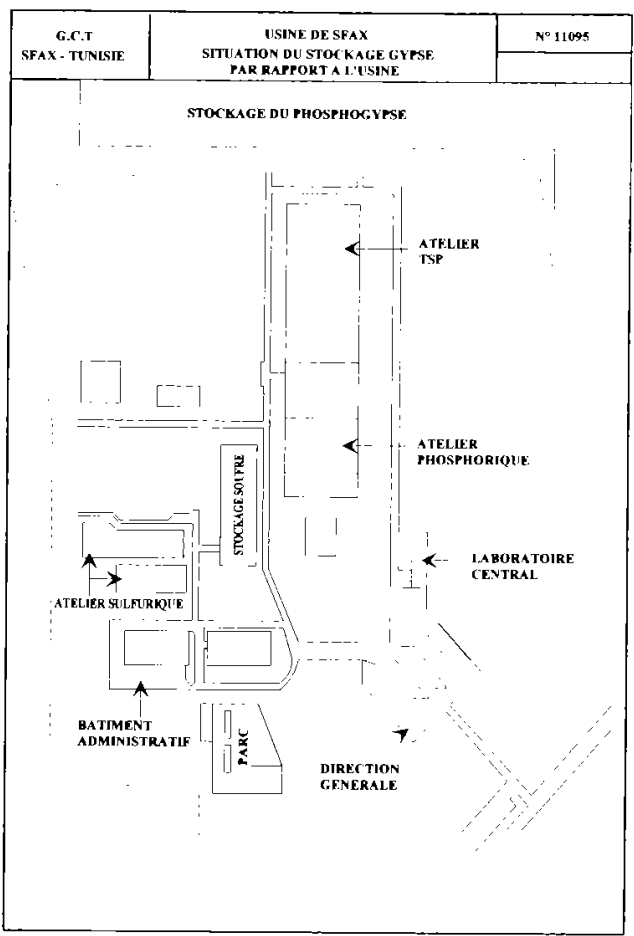

Fig. 1 .

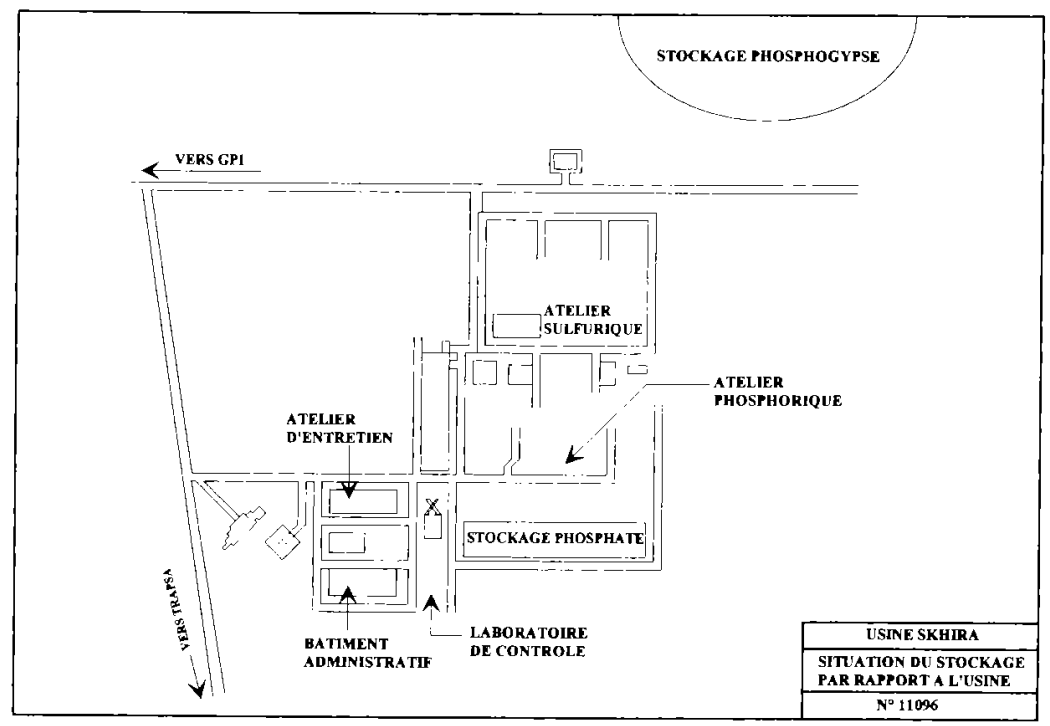

Fig. 2. 


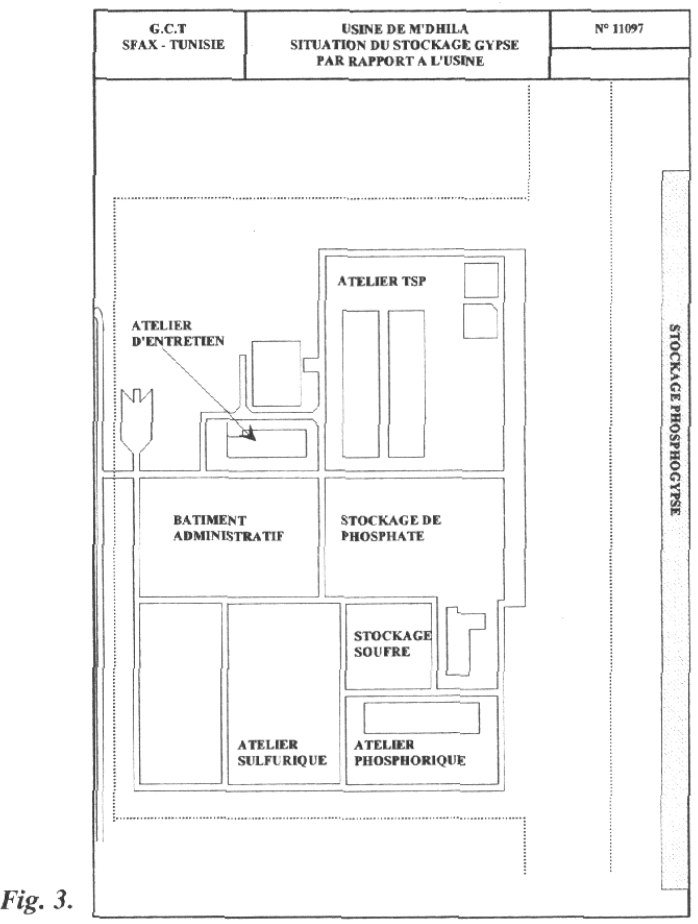

TABLEAU I

Résultats de mesure de l'activité pondérale en ${ }^{226} \mathrm{Ra}$ et de calcul du débit de dose absorbée dans l'air à un mètre.

Mean concentration results of ${ }^{226} \mathrm{Ra}$ and absorbed dose rate calculation at one metre in the air.

\begin{tabular}{|lccc|}
\hline Échantillons & Phosphate lavé & Acide phosphorique & Phosphogypse \\
\hline${ }^{226} \mathrm{Ra}\left(\mathrm{Bq} \mathrm{kg}^{-1}\right)$ & $460 \pm 80$ & $40 \pm 16$ & $350 \pm 70$ \\
$\begin{array}{l}\text { Débit de dose absorbée dans l'air } \\
\left(\mathrm{nGy} \mathrm{h}{ }^{-1}\right) \text { à un mètre de la surface }\end{array}$ & $212.1 \pm 36,9$ & $18.5 \pm 7,4$ & $161,4 \pm 32,3$ \\
\hline
\end{tabular}

* Coefficient de conversion en débit de dose pour le ${ }^{226} \mathrm{Ra}_{\text {en }} \mathrm{nGy} \mathrm{h} \mathrm{h}^{-1} / \mathrm{Bq} \mathrm{kg}^{-1}=0,461(5)$.

Le calcul du débit de dose absorbé dans l'air à $1 \mathrm{~m}$ dû aux radioéléments présents dans chaque produit est fonction de son activité massique.

- Le débit de dose absorbé dans l'air à un mètre du phosphogypse est de : $161,4 \mathrm{nGy} \mathrm{h}^{-1}$.

- Le coefficient de conversion retenu pour traduire le débit de dose absorbée en dose efficace dans l'air est de : 0,7 Sv Gy ${ }^{-1}$ pour les adultes (UNSCEAR,1993). 
- Le nombre d'heures de travail pour un agent de l'usine par an est de : 1760 heures ( 160 heures par mois $\times 11$ mois de travail).

- Le débit de dose absorbé dans l'air à un mètre des phosphates lavés est de : $212,1 \mathrm{nGy} \mathrm{h}^{-1}$.

- La dose équivalente totale due au ${ }^{226} \mathrm{Ra}$ pour un agent de l'usine qui passe un temps équivalent sur les phosphates et sur le phosphogypse est alors :

$0,7 \mathrm{~Sv} \mathrm{~Gy}^{-1} \times(212,1+161,4) \times 0,5 \mathrm{~Gy} \mathrm{~h}^{-1} \times 10^{-9} \times 1760 \mathrm{~h} \mathrm{an}^{-1}=0,23 \mathrm{mSv}^{-1}$ cependant il faut évaluer la dose due à l'inhalation du radon dans l'environnement du traitement de phosphate.

\section{Résultats de mesure des activités volumiques en radon 222 dans l'air}

La quantité de radon 222 produite est directement proportionnelle à la teneur du phosphate en ${ }^{226} \mathrm{Ra}$. Cependant, seule une fraction réussit à échapper du réseau, la majorité des atomes produits y restent prisonniers où ils se désintègrent. Selon la porosité du milieu, l'humidité, on obtient un taux d'exhalaison très variable. Une fois dans l'air extérieur, le radon est dispersé en fonction des conditions atmosphériques (Schmid et al.,1997).

Nous avons évalué la concentration de radon dans les différentes usines de la région de Sfax, M'dilla, Skhira et leurs environnements en exposant le dosimètre film LR 115 ; il s'agit d'un détecteur de particules alpha ouvert fabriqué par KODAK, qui se présente sous la forme d'un petit boîtier noir en plastique dont le couvercle sert de support à un film rouge, une fois le boîtier ouvert, le film est exposé directement à l'air ambiant. Il mesure sans discrimination les particules alpha émises par le radon et ses descendants la connaissance du facteur d'équilibre entre le radon et ses descendants est nécessaire pour estimer l'activité volumique du radon. Ce facteur peut varier d'un local à un autre dans les usines en raison de la composition en aérosols de l'atmosphère. Le détecteur est placé à une hauteur de un mètre à l'abri du soleil sur un support. Chaque point de mesure défini à l'intérieur des locaux de chaque usine et à ses environnements (Figs. 1 à 3) a fait l'objet de deux mesures à deux périodes différentes de l'année, la durée de l'exposition est de un mois. Après exposition le dosimètre est envoyé pour lecture dans le laboratoire Dosirad qui estime l'activité volumique avec un facteur de 0,4 . Les résultats obtenus sont mentionnés dans les tableaux II, III et IV.

D’après les résultats mentionnés aux tableaux II, III et IV, nous pouvons identifier les lieux qui peuvent présenter un risque «radon» potentiel :

- à la surface des tabias du phosphogypse, la concentration du radon est importante, cependant le risque d'irradiation deviendrait significatif dans le cas où des habitations seraient construites sur un dépôt de phosphogypse ; 
- près des filtres d'acides phosphoriques il y a un défaut d'aération ce qui explique une accumulation de radon relativement élevée dans ces lieux ;

- dans le bâtiment à l'intérieur des usines (salles de contrôle des ateliers d'acides phosphoriques et de trisuperphosphate, bureaux et laboratoires), la concentration en radon est toujours inférieure à $400 \mathrm{~Bq} \mathrm{~m}^{-3}$;

- aux voisinages des usines et à quelques kilomètres de l'usine (2 à $3 \mathrm{~km}$ ) la concentration en radon est en moyenne de $50 \mathrm{~Bq} \mathrm{~m}^{-3}$, cette valeur se trouve à l'intérieur des moyennes mondiales mesurées sous différents climats et conditions atmosphériques (10 à plus de 100 Bq m³) (Robé, 1992).

\section{TABLEAU II}

Résultats de mesure des activités volumiques moyennes en radon 222 dans l'usine de Sfax et son environnement.

Mean concentration results of radon 222 in the factory of Sfax and its environments.

\begin{tabular}{|c|c|c|c|}
\hline Lieu & $\begin{array}{c}\text { Date } \\
\text { d'exposition }\end{array}$ & $\begin{array}{l}\text { Concentration } \\
\text { moyenne }\left(\mathrm{Bq} \mathrm{m}^{-3}\right)\end{array}$ & $\begin{array}{l}\text { Erreur } \\
\text { en } \%\end{array}$ \\
\hline Salle de contrôle de l'atelier sulfurique & $\begin{array}{c}\text { Mai } 94 \\
\text { Décembre } 95\end{array}$ & 30 & 15 \\
\hline Atelier phosphorique à côté du filtre & $\begin{array}{c}\text { Mai } 94 \\
\text { Décembre } 94 .\end{array}$ & 1785 & 5 \\
\hline Salle de contrôle de l'atelier phosphorique & $\begin{array}{c}\text { Mai } 94 \\
\text { Décembre } 94\end{array}$ & 110 & 10 \\
\hline Bureau dans le bâtiment administratif & $\begin{array}{c}\text { Mai } 94 \\
\text { Décembre } 94\end{array}$ & 43 & 15 \\
\hline Salle de contrôle atelier TSP & $\begin{array}{c}\text { Mai } 94 \\
\text { Décembre } 94\end{array}$ & 69 & 11 \\
\hline Laboratoire central & $\begin{array}{c}\text { Mai } 94 \\
\text { Décembre } 94\end{array}$ & 156 & 8 \\
\hline Direction générale à $0,5 \mathrm{~km}$ de l'usine & $\begin{array}{c}\text { Mai } 94 \\
\text { Décembre } 94\end{array}$ & 36 & 15 \\
\hline Route de Soukra à $3 \mathrm{~km}$ de l'usine & $\begin{array}{c}\text { Mai } 94 \\
\text { Décembre } 94\end{array}$ & 23 & 16 \\
\hline Route de Gabés à $2 \mathrm{~km}$ de l'usine & $\begin{array}{c}\text { Avril } 94 \\
\text { Novembre } 95\end{array}$ & 48 & 14 \\
\hline Stockage de phosphate à $1 \mathrm{~m}$ de la surface & $\begin{array}{c}\text { Avril } 94 \\
\text { Novembre } 95\end{array}$ & 180 & 7 \\
\hline Tabia de phosphogypse à la surface & $\begin{array}{l}\text { Avril } 94 \\
\text { Mai } 95\end{array}$ & 3604 & 3 \\
\hline Tabia de phosphogypse à $1 \mathrm{~m}$ de la surface & $\begin{array}{l}\text { Avril } 94 \\
\text { Mai } 95\end{array}$ & 55 & 14 \\
\hline
\end{tabular}




\section{EXPOSITION AU RADON 222 DANS LES PHOSPHATES}

\section{TABLEAU III}

Résultats de mesure des activités volumiques moyennes en radon 222 dans l'usine de Skhira et son environnement.

Mean concentration results of radon 222 in the factory of Skhira and its Environments.

\begin{tabular}{|c|c|c|c|}
\hline Lieu & $\begin{array}{l}\text { Date } \\
\text { d'exposition }\end{array}$ & $\begin{array}{l}\text { Concentration } \\
\text { moyenne }\left(\mathrm{Bq} \mathrm{m}^{-3}\right)\end{array}$ & $\begin{array}{l}\text { Erreur } \\
\text { en } \%\end{array}$ \\
\hline Laboratoire de contrôle & $\begin{array}{c}\text { Juin } \\
\text { Octobre } 95\end{array}$ & 19 & 16 \\
\hline Salle de contrôle de l'atelier sulfurique & $\begin{array}{c}\text { Juin } 94 \\
\text { Octobre } 95\end{array}$ & 20 & 17 \\
\hline Salle de contrôle de l'atelier phosphorique & $\begin{array}{c}\text { Juin } 94 \\
\text { Octobre } 95\end{array}$ & 15 & 19 \\
\hline Atelier phosphorique à côté du filtre & $\begin{array}{c}\text { Juin } 94 \\
\text { Octobre } 95\end{array}$ & 284 & 12 \\
\hline Port de chargement & $\begin{array}{c}\text { Juin } 94 \\
\text { Octobre } 95\end{array}$ & 40 & 15 \\
\hline Aéroport à $5 \mathrm{~km}$ de l'usine & $\begin{array}{c}\text { Juin } 94 \\
\text { Octobre } 95\end{array}$ & 100 & 9 \\
\hline Stockage de phosphate à $1 \mathrm{~m}$ de la surface & $\begin{array}{c}\text { Juin } 94 \\
\text { Octobre } 95\end{array}$ & 89 & 18 \\
\hline Stockage de phosphate à la surface & $\begin{array}{c}\text { Juin } 94 \\
\text { Octobre } 95\end{array}$ & 318 & 8 \\
\hline $\begin{array}{l}\text { Tabia de phosphogypse au milieu } \\
\text { du terril à la surface }\end{array}$ & $\begin{array}{c}\text { Juin } 94 \\
\text { Octobre } 95\end{array}$ & 2736 & 4 \\
\hline $\begin{array}{l}\text { Tabia de phosphogypse au milieu } \\
\text { du terril à } 1 \mathrm{~m}\end{array}$ & $\begin{array}{c}\text { Juin } 94 \\
\text { Octobre } 95\end{array}$ & 30 & 13 \\
\hline
\end{tabular}

\section{Estimation de l'énergie alpha potehtielle volumique}

\subsection{Energie alpha potentielle (EAP)}

L'énergie alpha potentielle (EAP) due aux descendants à vie courte du radon 222 est définie par la somme des énergies des particules alpha des descendants du radon émises lorsque tous les produits de filiation à vie courte se sont désintégrés.

Elle s'exprime en joules (J) dans le système SI. Elle peut aussi être exprimé en méga électronvolts $(\mathrm{MeV}) .1 \mathrm{eV}=1,6 \times 10^{-19} \mathrm{~J}$.

\subsection{Energie alpha potentielle volumique (EAPv)}

Il s'agit de l'énergie alpha potentielle précédemment définie rapportée à l'unité de volume. Elle s'exprime généralement en joules par mètre cube $\left(\mathrm{J} \mathrm{m}^{-3}\right)$. 


\section{TABLEAU IV}

Résultats de mesure des activités volumiques moyennes en radon 222 dans l'usine de M'dilla et son environnement.

Mean concentration results of radon 222 in the factory of M'dilla and its environment.

\begin{tabular}{|l|ccc|}
\hline Lieu & $\begin{array}{c}\text { Date } \\
\text { d'exposition }\end{array}$ & $\begin{array}{c}\text { Concentration } \\
\text { moyenne }\left(\mathbf{B q} \mathbf{~ m}^{-3}\right)\end{array}$ & $\begin{array}{c}\text { Erreur } \\
\text { en } \%\end{array}$ \\
\hline Salle de contrôle de l'atelier sulfurique & $\begin{array}{c}\text { Mai } 94 \\
\text { Mars } 95\end{array}$ & 64 & 12 \\
Salle de contrôle de l'atelier phosphorique de l'atelier TSP & $\begin{array}{c}\text { Mai } 94 \\
\text { Mars } 95\end{array}$ & 37 & 15 \\
\hline Atelier phosphorique à côté du filtre & $\begin{array}{c}\text { Mai } 94 \\
\text { Mars } 95\end{array}$ & 53 & 14 \\
\hline Bâtiment administratif - bureau & Mai 94 & 720 & 8 \\
\hline À 2 km de l'usine et à 1 m du sol & Mars 95 & 71 & 11 \\
\hline Stockage de phosphate à la surface & Mai 94 & 48 & 14 \\
\hline Tabia de phosphogypse à la surface & Mars 95 & & 10 \\
\hline Tabia du phosphogypse à 1 m de la surface & Mai 94 & 318 & 3 \\
\hline
\end{tabular}

La radioactivité provenant du traitement de phosphate devrait être gérée conformément aux recommandations de la Commission internationale de protection radiologique (CIPR) pour des pratiques (ICPR 60, 1990).

Pour le risque radon, c'est l'énergie alpha potentielle absorbée par an qui est considérée comme indicateur de risque (ICPR 65, 1993). La CIPR 65 recommande une limite annuelle d'exposition pour les travailleurs égale à $14 \mu \mathrm{J} \mathrm{m}^{-3} \mathrm{~h}$ en moyenne sur 5 ans, sans dépasser $35 \mathrm{~mJ} \mathrm{~h} \mathrm{~m}^{-3}$ par an. En tenant compte du nombre d'heures de travail de 1760 par an, ces limites d'expositions correspondent en terme d'énergie alpha potentielle à une limite annuelle de $8 \mathrm{~mJ} \mathrm{~m}^{-3}$ en moyenne sans dépasser $20 \mu \mathrm{J} \mathrm{m}^{-3}$ par an.

Nous avons évalué l'énergie alpha potentielle volumique en tenant compte du facteur d'équilibre qui est défini comme étant le rapport entre l'énergie alpha potentielle volumique d'un mélange de descendants du radon et l'énergie alpha 
potentielle volumique relative au mélange s'il était à l'équilibre avec le radon (Milu, 1996).

Pour cela, on considère que le radon avec ses descendants est en équilibre dans les usines et leur environnements avec un facteur d'équilibre $(F)$ variable défini par la relation suivante :

$$
F=\frac{\text { 178. EAP }\left(\mu \mathrm{J} \mathrm{m}^{-3}\right)}{C_{R n}\left(\mathrm{~Bq} \mathrm{~m}^{-3}\right)}
$$

$C_{R n}\left(\mathrm{~Bq} \mathrm{~m}^{-3}\right)$ étant la concentration du radon 222.

En l'absence d'une évaluation du facteur d'équilibre en chaque point de mesure, on a fait une comparaison des concentrations moyennes en gaz radon avec la valeur correspondant à la limite d'EAPV de la CIPR 65 pour un facteur de 0,4 , un débit respiratoire du travailleur standard de $1,2 \mathrm{~m}^{3} \mathrm{~h}^{-1}$ d'air et 2000 heures de travail par an.

Les résultats de cette comparaison sont mentionnés dans les tableaux V, VI et VII.

\section{TABLEAU V}

Pourcentage de la limite de l'EAPv recommandée pour les travailleurs dans l'usine de Sfax et son environnement.

PAEv limit percentage recommended for the worker in factory of Sfax and its environnement.

\begin{tabular}{|c|c|c|c|}
\hline Lieu & $\begin{array}{l}\text { EAP moyenne } \\
\left(\mu \mathrm{J} \mathrm{m}^{-3}\right)\end{array}$ & $\begin{array}{l}\% \text { de la limite } \\
\text { d'EAP de } \\
\text { la CIPR } 65\end{array}$ & $\begin{array}{l}\text { Dose efficace } \\
\text { mSv par an }\end{array}$ \\
\hline Salle de contrôle de l'atelier sulfurique & 0,067 & 0,8 & 0,16 \\
\hline Atelier phosphorique à côté du filtre & 4,011 & 50,1 & 9,9 \\
\hline Salle de contrôle de l'atelier phosphorique & 0,247 & 3,1 & 0,61 \\
\hline Bureau dans le bâtiment administratif & 0,096 & 1,2 & 0,24 \\
\hline Salle de contrôle atelier TSP & 0,155 & 1,9 & 0,38 \\
\hline Laboratoire central & 0,350 & 4,3 & 0,86 \\
\hline Direction générale à $0,5 \mathrm{~km}$ de l'usine & 0,14 & 1 & 0,34 \\
\hline Route de Soukra à $3 \mathrm{~km}$ de l'usine & 0,089 & 0,64 & 0,21 \\
\hline Route de Gabés à 2 km de l'usine & 0,196 & 1,3 & 0,48 \\
\hline Stockage de phosphate à $1 \mathrm{~m}$ de la surface & 0,397 & 1,34 & 0,69 \\
\hline Tabia de phosphogypse à la surface & 8,09 & 101 & 19,9 \\
\hline Tabia de phosphogypse à $1 \mathrm{~m}$ de la surface & 0,123 & 1,54 & 0,3 \\
\hline
\end{tabular}




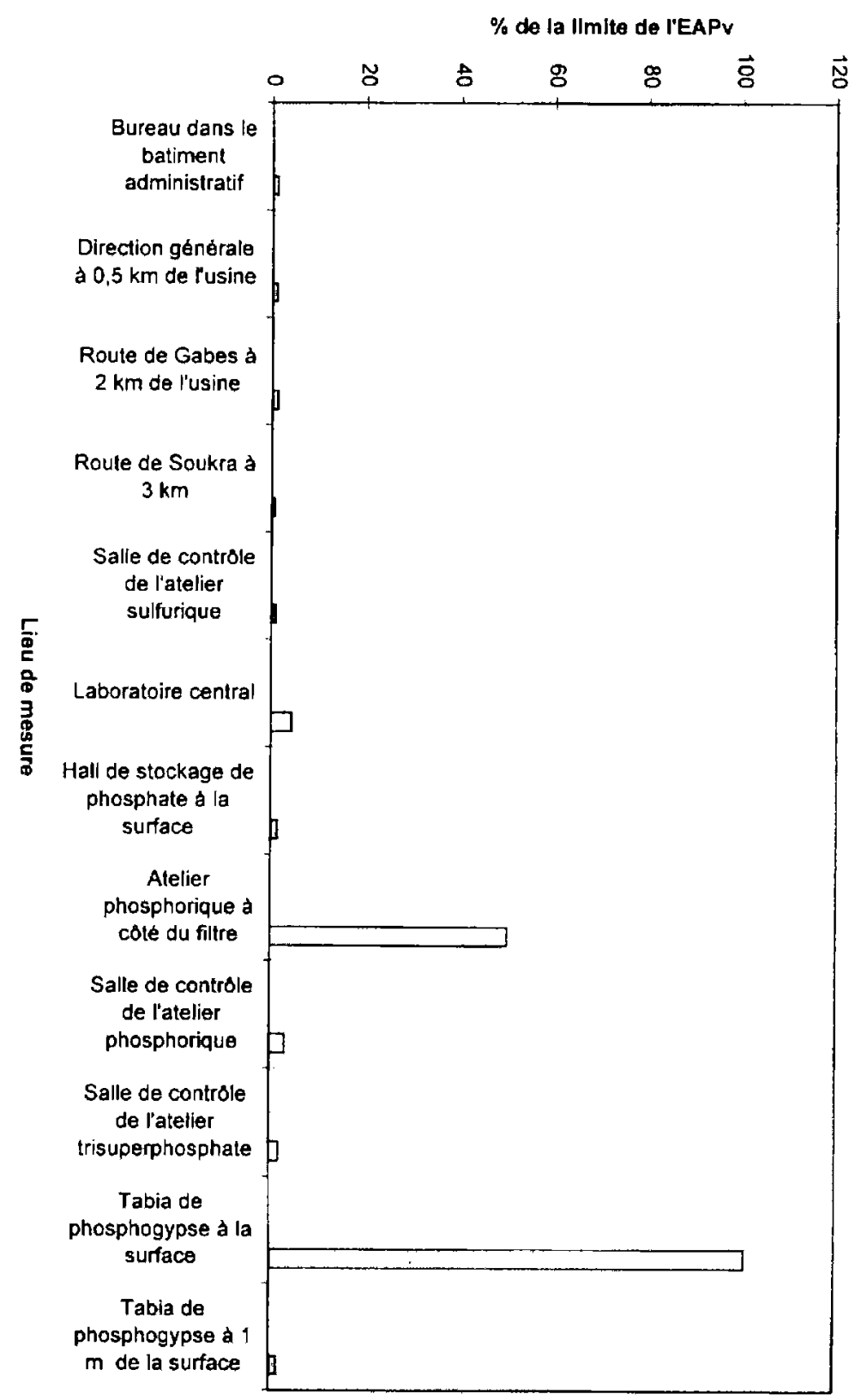

Fig. 4. - Variation du \% de la limite d'EAPv dans l'usine de Sfax.

$\%$ PEAv limit's variation in Sfax factory. 


\section{TABLEAU VI}

Pourcentage de la limite de l'EAPv recommandée pour les travailleurs dans l'usine de Skhira et son environnement.

PAEv limit percentage recommended for the worker in factory of Skhira and its environnement.

\begin{tabular}{|lccc|}
\hline Lieu & $\begin{array}{c}\text { EAP moyenne } \\
\left(\boldsymbol{\mu J} \mathbf{~ m}^{-3}\right)\end{array}$ & $\begin{array}{c}\text { \% de la limite } \\
\text { d'EAP de } \\
\text { la CIPR 65 }\end{array}$ & $\begin{array}{c}\text { Dose efficace } \\
\text { mSv par an }\end{array}$ \\
\hline Laboratoire de contrôle & 0,042 & 0,53 & 0,10 \\
\hline Salle de contrôle atelier sulfurique & 0,05 & 0,56 & 0,12 \\
\hline Salle de contrôle atelier phosphorique & 0,033 & 0,42 & 0,08 \\
\hline Atelier phosphorique à côté du filtre & 0,34 & 7,97 & 0,84 \\
\hline Port de chargement & 0,016 & 1,1 & 0,039 \\
\hline Aéroport à 5 km de l'usine & 0,224 & 2,8 & 0,55 \\
\hline Hall de stockage de phosphate à 1 m du sol & 0,022 & 2,5 & 0,054 \\
\hline Hall de stockage de phosphate à la surface & 1,667 & 8,9 & 4,11 \\
\hline $\begin{array}{l}\text { Tabia de phosphogypse au milieu } \\
\text { de terril à la surface }\end{array}$ & 6,12 & 76,8 & 15,1 \\
\hline Tabia de phosphogypse à 1 m de la surface & 0,0067 & 0,8 & 0,16 \\
\hline
\end{tabular}

\section{TABLEAU VII}

Pourcentage de la limite de l'EAPv recommandée pour les travailleurs dans l'usine de M'dhilla et son environnement.

PAEv limit percentage recommended for the worker in factory of M'dhilla and its environnement.

\begin{tabular}{|lccc|}
\hline Lieu & $\begin{array}{c}\text { EAP moyenne } \\
\left(\mu \mathbf{J} \mathbf{~ m}^{-3}\right)\end{array}$ & $\begin{array}{c}\text { \% de la limite } \\
\text { d'EAP de } \\
\text { la CIPR } \mathbf{6 5}\end{array}$ & $\begin{array}{c}\text { Dose efficace } \\
\mathbf{m S v} \text { par an }\end{array}$ \\
\hline $\begin{array}{l}\text { Salle de contrôle atelier sulfurique } \\
\text { Salle de contrôle atelier TSP }\end{array}$ & 0,143 & 1,8 & 0,35 \\
\hline Salle de contrôle atelier phosphorique & 0,083 & 1 & 0,20 \\
\hline Atelier phosphorique à côté du filtre & 0,119 & 1,4 & 0,29 \\
\hline Bâtiment administratif à côté du filtre & 1,246 & 20,2 & 3,07 \\
\hline À 2 km de l'usine et à I m du sol & 1,159 & 1,9 & 2,86 \\
\hline Stockage de phosphate à la surface & 0,187 & 1,3 & 0,46 \\
\hline Tabia de phosphogypse au milieu & 0,714 & 8,9 & 1,76 \\
\hline Tabia de phosphogypse à 1 m de la surface & 8,63 & 108 & 21,26 \\
\hline
\end{tabular}


H. MAHJOUBI et al.

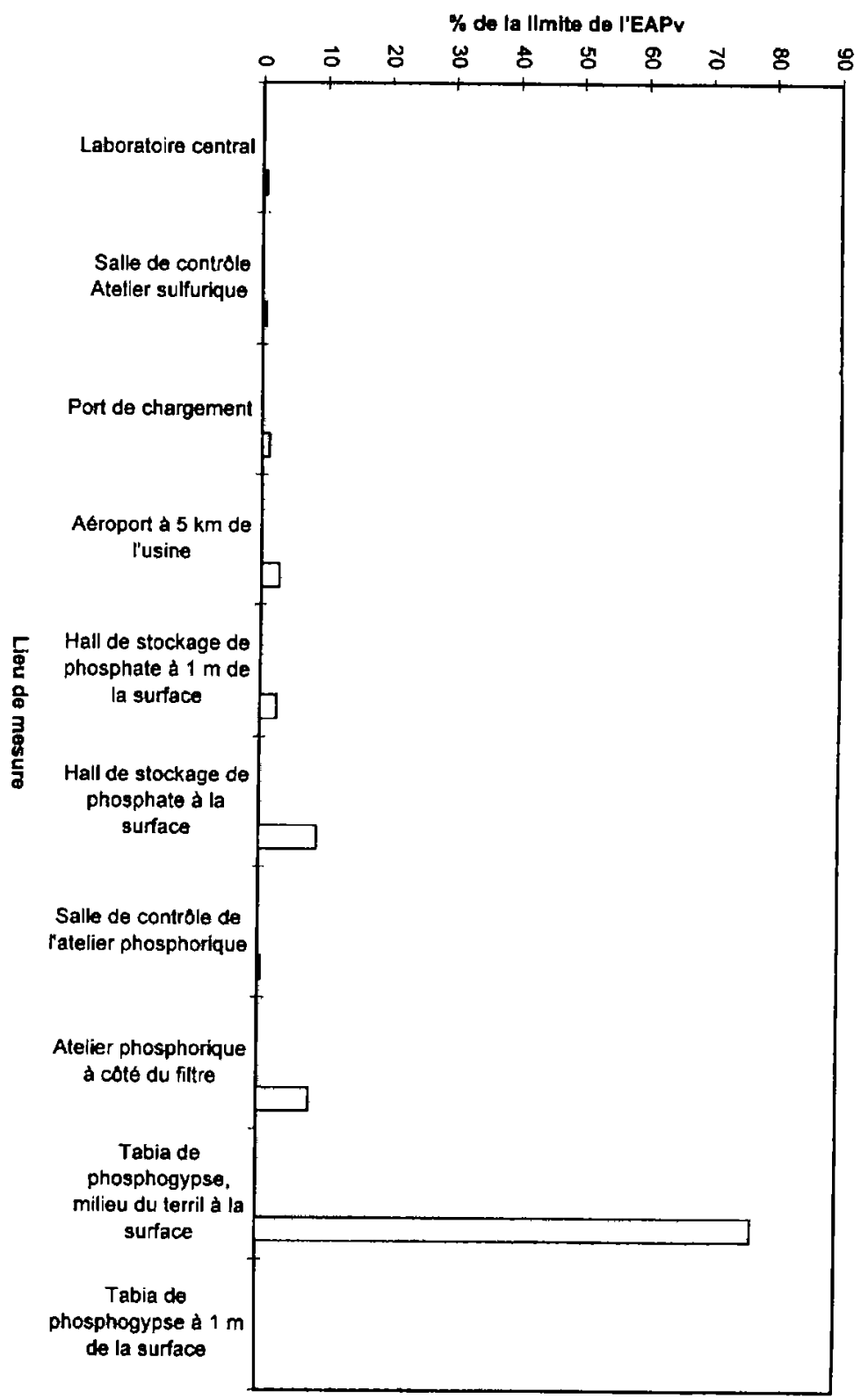

Fig. 5. - Variation du \% de la limite d'EAPv dans l'usine de S'Khira.

\% PEAv limit's variation in S'Khira factory. 


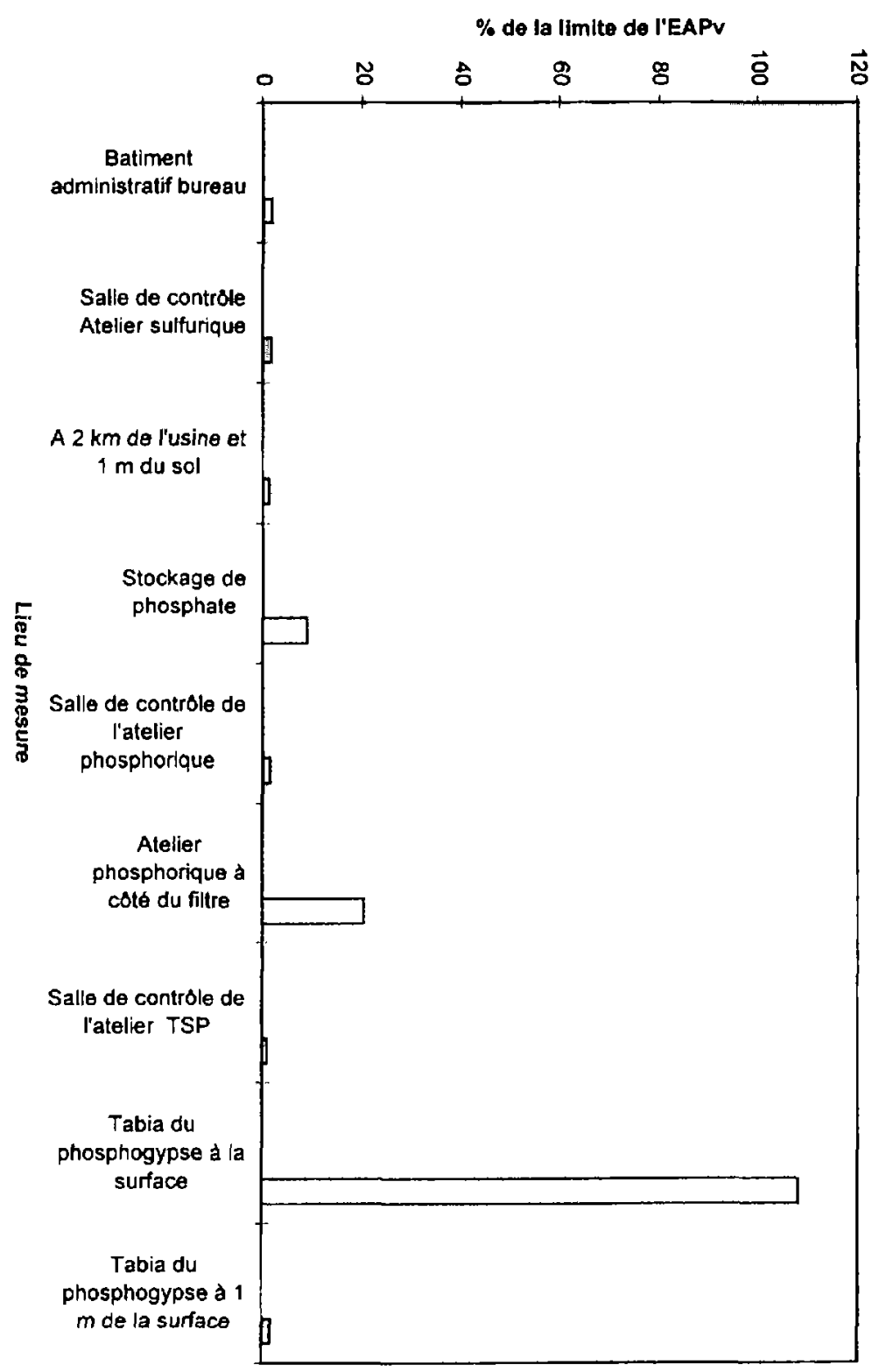

Fig. 6. - Variation du \% de la limite d'EAPv dans l'usine de M'Dhila.

\% PEAv limit's variation in M'Dhila factory. 


\section{Interprétation des résultats}

Les tableaux V à VII mettent en évidence une forte contribution à l'énergie alpha potentielle volumique dans les ateliers phosphoriques près des filtres et dans les tabias de phophogypse à la surface, on note aussi qu'une valeur de 0,8 pour le facteur d'équilibre ne permet plus le respect de la limite d'EAPv de la CIPR 65 pour une présence permanente dans l'atelier phosphorique de l'usine de Sfax.

En se plaçant dans les conditions les plus défavorables où le facteur d'équilibre est de 0,8 , on constate que l'exposition relative aux différents points de mesure à l'intérieur des locaux des usines reste inférieure aux recommandations de la CIPR sauf au niveau de l'atelier phosphorique à coté du filtre de l'usine de Sfax, ce qui conduit à un risque d'excès d'effets sanitaires graves par année d'exposition égal à $8 \times 1760 \times 8 \times 10^{-5}=1,12 \times 10^{-3}$, par application du facteur de risque de $8 \times 10^{-5}$ par $\mathrm{mJ} \mathrm{m}^{-3} \mathrm{~h}$ retenu par la publication CIPR 65. Après 10 ans d'exposition au niveau du seuil proposé le risque pour un agent de l'usine travaillant dans les tabias de phosphogypse est de l'ordre de $1,1 \%$.

À 1 mètre de la surface des tabias de phosphogypse, l'énergie alpha potentielle moyenne varie entre 0,10 et $0,24 \mathrm{~mJ} \mathrm{~m}^{-3} \mathrm{~h}$, le niveau d'exposition diminue rapidement par rapport à celui mesurée à la surface, dont la valeur est à la limite moyenne recommandée par la CIPR 65 pour les travailleurs.

Le calcul de l'exposition externe rajoutée à l'exposition due au radon 222 est toujours inférieure à $20 \mathrm{mSv}$, limite recommandée par la CIPR 60 .

\section{Conclusion}

L'analyse des données montre qu'il y a une variabilité de concentration de radon dans les différents points de mesure. En effet le radon est un gaz, sa concentration en un point dépend de la position de la source de radon par rapport au point de mesure. Par ailleurs le niveau moyen du radon mesuré dans les usines est faible et reste acceptable.

Cette étude nous a permis de conclure que la somme de l'exposition externe et de l'exposition due au radon pour les travailleurs est inférieur à $20 \mathrm{mSv}$ par an.

Elle nous a permis également d'orienter la suite des mesures à réaliser, en particulier, il est nécessaire d'évaluer le facteur d'équilibre dans tout point de mesure en vue d'une évaluation plus réaliste des expositions et d'une réelle démonstration du respect de la limite en énergie alpha potentielle volumique de la CIPR 65 et de l'optimisation des expositions résiduelles. 


\section{RÉFÉRENCES}

ICRP Publication 60 (1990) Recommendations of the International Commission on Radiation Protection, Ann. ICRP, 21 (1-3).

ICRP Publication 65 (1993) Protection Against radon-222 at Home and at Work, Ann. ICRP, 23 (2).

Milu C. (1996) Qualitative correlations between some influencing factors and radon and thoron concentrations in romanian houses. In: Radon in Human Environment, IAEA, RC-J1, ROM-7717/RB.

Robé M.-Ch., Rannou A., Le Bronec J., Tymen G. (1992) Le radon dans les habitations : identification des voies de transfert et caractérisation des aérosols radioactifs produits, Mém. Expl, Cartes géologiques et minières de la Belgique, $n^{\circ} 32,93-130$.

Robé M.-Ch., Rannou A., Le Bronec J. (1992) Radon Measurements in the Environment in France, Radiat. Prot. Dosim., 45, 455-457.

Schmid S., Wiegand J.(1997) The influence of traffic vibrations on the radon potential, Health Physics., 74, 231-236.

UNSCEAR (1993) Ionizing Radiation Sources and Biological Effects. Report to General Assembly with Annexes, UNSCEAR Publications, New York. 